\title{
Trajectory Control of a Quadrotor Using a Control Allocation Approach
}

\author{
Hammad Zaki ${ }^{1}$, Mustafa Unel ${ }^{1,2}$ and Yildiray Yildiz ${ }^{3}$
}

\begin{abstract}
A quadrotor is an underactuated unmanned aerial vehicle with four inputs to control the dynamics. Trajectory control of a quadrotor is a challenging task and usually tackled in a hierarchical framework where desired/reference attitude angles are analytically determined from the desired command signals, i.e. virtual controls, that control the positional dynamics of the quadrotor and the desired yaw angle is set to some constant value. Although this method is relatively straightforward, it may produce large and nonsmooth reference angles which must be saturated and low-pass filtered. In this work, we show that the determination of desired attitude angles from virtual controls can be viewed as a control allocation problem and it can be solved numerically using nonlinear optimization where certain magnitude and rate constraints can be imposed on the desired attitude angles and the yaw angle need not be constant. Simulation results for both analytical and numerical methods have been presented and compared. Results for constrained optimization show that the flight performance is quite satisfactory.
\end{abstract}

\section{INTRODUCTION}

Nowadays Unmanned Aerial Vehicles (UAV) are utilized in many civilian and military applications. Quadrotor is one of the most used kinds of UAV because of its ability to complete difficult tasks due to its vertical take off and landing (VTOL) capability. Quadrotor is a highly nonlinear system, so trajectory tracking is a challenging task. In recent years, there have been a number of papers dealing with various problems inherent to the exploitation of quadrotors dynamics. Trajectory control of quadrotors has gained great interest in the unmanned air vehicle community. Dynamic modeling issues were addressed in [1] and [2]. Bouabdallah [1] used the linear model and compared the results for PID controller with LQ controller. The results showed that hovering control was satisfactory for PID controller but for stability in the presence of disturbances, results were not so efficient. Different nonlinear control techniques such as sliding mode control, backstepping control and feedback linearization were used [3]-[6]. The sliding mode approach provided average results to stabilize the attitude while the structural changes affected the control quality because of the high-frequency disturbances. Comparison of feedback linearization and adaptive sliding mode to estimate uncertainty such as ground effects and noisy conditions was presented in [7]. Madani [8] used the technique of dividing

\footnotetext{
${ }^{1}$ Faculty of Engineering and Natural Sciences, Sabanci University, Tuzla Istanbul, Turkey

${ }^{2}$ Integrated Manufacturing Technologies Research and Application Center, Sabanci University, Istanbul, Turkey

$\{$ hammadzaki, munel\}@sabanciuniv.edu

${ }^{3}$ Department of Mechanical Engineering, Bilkent University, Cankaya, Ankara, Turkey

yyildiz@bilkent.edu.tr
}

quadrotor dynamics into many linearly connected subsystems and proposed a full-state backstepping and sliding mode control technique based on the Lyapunov stability theory for quadrotor to track the desired trajectories. Adaptive fuzzy backstepping technique was used [9], in which a control law for the model was generated by fuzzy system using backstepping approach. Robust trajectory tracking was presented which used combination of integral backstepping and PID controller to stabilize the dynamics [10]. Global trajectory tracking control [11] was proposed without linear velocity measurements but efficiency in terms of position errors was not so good. Flatness-based control of a quadrotor via feedback linearization was performed in [12]. $H_{\infty}$ and model predictive control were used to solve the problem of trajectory control [13]. Design and development of a tilt-wing quadrotor and its robust control using a hierarchical structure were considered in [14]-[16]. It consists of two parts: upper level control for positional dynamics which generates virtual controls and lower level control which provides tracking of desired attitude angles computed from virtual controls. Desired or reference angles were obtained through analytical formulas. More recently adaptive nonlinear hierarchical control of a tilt-wing quadrotor was developed in [17] and [18]. Chan also applied hierarchical control where motor speed control and attitude control were utilized [19]. Optimized trajectory planning algorithms had been applied using nonlinear optimization and results were shown for linear path where desired angles were obtained through analytical method [20].

In this work, a control allocation type technique is formulated on the nonlinear dynamics of the quadrotor to obtain optimum values for the desired attitude angles from command signals (virtual controls) developed for positional dynamics. Different from an existing study [21] where control allocation approach is applied to distribute the total control effort among real actuators optimally, here the command signals are utilized to get the values for desired attitude angles. With the help of hierarchical control, controller design is divided into two parts: position control and attitude control. Positional dynamics of the quadrotor is considered as the underdetermined part as it contains more unknown variables than the equations. In control allocation, nonlinear constraint optimization is used to obtain required actuator inputs according to command signals by solving an underdetermined system [22]. In our approach, high level controller is designed to obtain the desired command signals from the positional dynamics. Nonlinear constrained optimization is used to get desired attitude angles $\left(\phi_{r}, \theta_{r}, \psi_{r}\right)$ according to the command signals. Sequential quadratic programming $(S Q P)$ algorithm is used in nonlinear constraint optimiza- 
tion. Low level controllers are implemented to ensure that the attitude angles are adjusted according to desired trajectory. Analytical method for the calculation of reference angles is also applied and results are shown for the same trajectories. Simulation results for both analytical and numerical methods have been presented for comparison to show the efficiency of the proposed technique.

The organization of the paper is as follows: Section II outlines the dynamics of the quadrotor. Section III presents the control allocation and nonlinear optimization. Section IV presents the controller design. Section V and VI present the simulation results and the summary of the paper respectively.

\section{QUADROTOR DYNAMICS}

The quadrotor is a type of aerial robot which consists of four rotors separated in a cross structure. The crossed configuration shows robustness even the mechanically linked motors are heavier than the frame [23]. Reduction gears are used to connect propellers to the motors. The motion of the quadrotor depends upon the direction of the rotation of the propellers of rotors. Front and rear propellers rotate counter clockwise, while the left and the right ones turn clockwise. There is no need of the tail rotor because of opposite pairs directions configuration unlike the standard helicopter structure. Fig. 1 shows the model in a hovering state, where all the propellers have the same speed. The motion of a

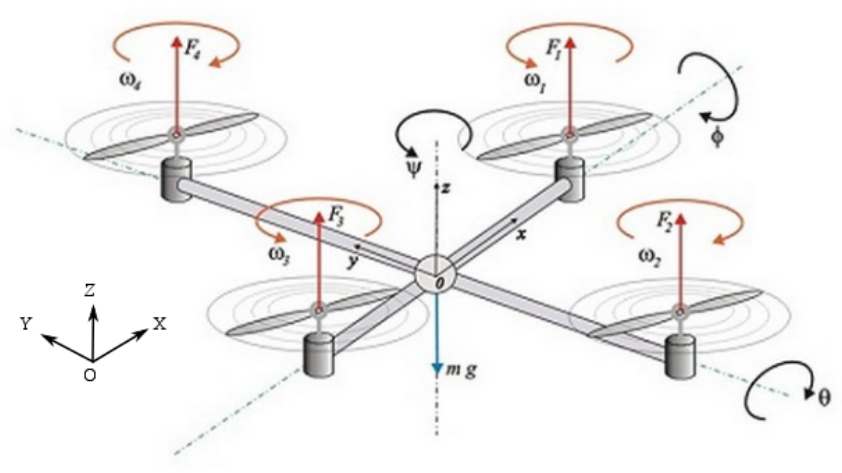

Fig. 1: Quadrotor Dynamics (Adapted from [24])

quadrotor is described by two frame of references, one of which is fixed and called inertial frame and the other one which is moving, called body frame. The vertical force w.r.t body-fixed frame is provided by thrust command $\left(U_{1}\right)$ which is used to raise (or lower) the quadrotor by increasing and decreasing the speed of the propellers equally respectively. Similarly, increasing (or decreasing) the left propeller speed and decreasing (or increasing) the right one results into roll command $\left(U_{2}\right)$, which makes the quadrotor to turn due to the torque with respect to the $x$ axis. Pitch command $\left(U_{3}\right)$ is very similar to the roll but in this case, increase (or decrease) in the rear propeller speed and decrease (or increase) in the front one leads to a torque with respect to the $y$ axis which makes the quadrotor to turn. In order to enable the quadrotor to turn along $z$ axis, torque is provided by the yaw command $\left(U_{4}\right)$ which is provided by increasing (or decreasing) the front-rear propellers speed and by decreasing (or increasing) the speed of the left-right couple propellers. Detailed description of the quadrotor dynamics can be found in [23]. The quadrotor positional dynamics expressed in world frame and attitude dynamics expressed in body frame are given as

$$
\begin{aligned}
\ddot{X} & =(\sin \psi \sin \phi+\cos \psi \sin \theta \cos \phi) \frac{U_{1}}{m} \\
\ddot{Y} & =(-\cos \psi \sin \phi+\sin \psi \sin \theta \cos \phi) \frac{U_{1}}{m} \\
\ddot{Z} & =-g+(\cos \theta \cos \phi) \frac{U_{1}}{m} \\
\dot{p} & =\frac{I_{y y}-I_{z z}}{I_{x x}} q r-\frac{J_{p r o p}}{I_{x x}}+\frac{U_{2}}{I_{x x}} \\
\dot{q} & =\frac{I_{z z}-I_{x x}}{I_{y y}} p r+\frac{J_{p r o p}}{I_{y y}}+\frac{U_{3}}{I_{y y}} \\
\dot{r} & =\frac{I_{x x}-I_{y y}}{I_{z z}} p q+\frac{U_{4}}{I_{z z}}
\end{aligned}
$$

where $U_{1}, U_{2}, U_{3}$ and $U_{4}$ are defined as

$$
\begin{aligned}
& U_{1}=b\left(\omega_{1}^{2}+\omega_{2}^{2}+\omega_{3}^{2}+\omega_{4}^{2}\right) \\
& U_{2}=l b\left(-\omega_{2}^{2}+\omega_{4}^{2}\right) \\
& U_{3}=l b\left(-\omega_{1}^{2}+\omega_{3}^{2}\right) \\
& U_{4}=d\left(-\omega_{1}^{2}+\omega_{2}^{2}-\omega_{3}^{2}+\omega_{4}^{2}\right)
\end{aligned}
$$

where $\omega_{1}, \omega_{2}, \omega_{3}$ and $\omega_{4}$ are the speed of front, right, rear and left propellers; $l, b$ and $d$ are the length of the rotor arm, thrust factor and drag factor, respectively. First three equations represent the positional dynamics and last three equations describe the orientation of the quadrotor in (1).

The relationship between angular velocities of quadrotor in body frame $(\eta)$ and the Euler rates in world frame $(\Omega)$ is given by

$$
\eta=E(\Theta) \Omega
$$

where $E(\Theta)$ is the velocity transformation matrix defined as

$$
E(\Theta)=\left[\begin{array}{ccc}
1 & \sin \phi \tan \theta & \cos \phi \tan \theta \\
0 & \cos \phi & -\sin \phi \\
0 & \frac{\sin \phi}{\cos \theta} & \frac{\cos \phi}{\cos \theta}
\end{array}\right]
$$

where $\Theta=[\phi, \theta, \psi]^{T}, \Omega=[\dot{\phi}, \dot{\theta}, \dot{\psi}]^{T}$ and $\eta=[p, q, r]^{T}$.

\section{Control Allocation AND Nonlinear OPTIMIZATION}

\section{A. Control Allocation}

Motion control is used to control the motion of the mechanical systems. All mechanical systems require control forces which are produced by the end effectors. Actuators are the devices which are used to control these forces. Sometimes there are more actuators than required. In that case system becomes underdetermined due to more number of unknown variables than the number of equations. Control allocation approach is used for such underdetermined systems. The purpose of the control allocation is to distribute the desired 
control effort to all redundant actuators. In order to provide optimal solution in the presence of coupling among the actuators due to underdetermined nature, optimization techniques are used for this purpose. Control allocation is a hierarchical type algorithm which consists of the following three parts [25].

High level controller is used to produce virtual command inputs.

Optimization is used to distribute the total virtual command among the actuators through linear and nonlinear optimization depending upon the cost function to be minimized and constraints.

Low level controller is used to produce required force at each end effector with the help of actuators.

Control allocation algorithm is advantageous due to the following reasons.

1) It is used to solve problems like actuator position and rate saturation.

2) It is used to produce fault tolerant system in case of failure of any actuators.

3) It is used to produce size and cost effective mechanical design by choosing the optimal set of the actuators rather than the small number of the actuators.

In this work positional dynamics of the quadrotor is exploited to apply the control allocation approach. If we look at the positional dynamics of the quadrotor in (1), it consists of three equations and four unknown variables $\left(\phi, \theta, \psi, U_{1}\right)$, so it can be considered as underdetermined system. In [22] control allocation approach had been used to solve underdetermined system where nonlinear optimization problem had been formulated. As underdetermined part of the quadrotor consists of nonlinear equations, nonlinear optimization is required to get the optimal solution. The purpose of the control allocation is to generate command inputs that must be produced jointly by all actuators which in our case are $\phi, \theta, \psi$ and $U_{1}$. Optimization problem along with the nonlinear and linear constraints is formulated as

$$
J(\zeta)=\frac{1}{2}\left(s^{T} s\right)
$$

where $J(\zeta)$ is the cost function to be minimized and $s$ is a slack variable. In our problem $\zeta$ is given by:

$$
\zeta=\left[\phi, \theta, \psi, U_{1}\right]
$$

Cost function is minimized subject to the following nonlinear and linear constraints

$$
\begin{aligned}
& s=\tau-B(\zeta) \\
& \zeta_{\min } \leq \zeta \leq \zeta_{\max } \\
& \Delta \zeta \leq C
\end{aligned}
$$

where $\tau$ is the desired command inputs that is provided by the high level controller of the hierarchical control. $\tau$ and $B(\zeta)$ are given as

$$
\tau=\left[\begin{array}{lll}
\ddot{X} & \ddot{Y} & \ddot{Z}
\end{array}\right]^{T}
$$

$$
B(\zeta)=\left[\begin{array}{c}
(\sin \psi \sin \phi+\cos \psi \sin \theta \cos \phi) \frac{U_{1}}{m} \\
(-\cos \psi \sin \phi+\sin \psi \sin \theta \cos \phi) \frac{U_{1}}{m} \\
-g+(\cos \theta \cos \phi) \frac{U_{1}}{m}
\end{array}\right]
$$

$\zeta_{\min }$ and the $\zeta_{\max }$ are the constrained range for $\zeta$. Actuator rate constraint $\Delta \zeta$ is included in the formulation by limiting the change in the control inputs $\zeta$ from the last sampling instant to some constant $C$.

\section{B. Nonlinear Optimization}

Optimization deals with finding the feasible solution for $n$ variables to minimize or maximize any function. Nonlinear programming is a mathematical tool, used to minimize the cost function subject to linear and nonlinear constraints. Feasible regions show the set of optimized variables which lie in the range of constraints. In unconstrained optimization feasible solutions converge to some finite values. In constrained optimization, nonlinear problem is converted into many subspaces and then different algorithms are applied to obtain optimum solution according to constraints.

Sequential quadratic programming $(S Q P)$ is a nonlinear optimization tool which is one of the most effective iterative methods. Advantage of using $S Q P$ is to find good initial point for feasible solution. Active set method is used in $S Q P$. It converts nonlinear problem into the subspace based on Lagrange approximation. Lagrange function is given by:

$$
L(\zeta, \lambda)=J(\zeta)+\sum_{i=1}^{m} \lambda_{i} \cdot g_{i}(\zeta)
$$

where $g_{i}(\zeta)$ is a gradient and $\lambda_{i}$ is a Lagrange multiplier. Detailed description of $S Q P$ is given in [26], briefly it consists of following three parts:

Hessian Matrix part uses positive definite quasi Newton optimization method for Lagrange function using BroyedeFlecher-Golfarb-Shanon $(B F G S)$ [26] method at every instant $k$.

$$
H_{k+1}=H_{k}+\frac{q_{k} q_{k}^{T}}{q_{k}^{T} l_{k}}-\frac{H_{k} l_{k} l_{k}^{T} H_{k}}{l_{k}^{T} H_{k} l_{k}}
$$

where

$$
\begin{gathered}
l_{k}=\zeta_{k+1}-\zeta_{k} \\
q_{k}=\nabla J\left(\zeta_{k+1}\right)+\sum_{i=1}^{m} \lambda_{i} \cdot g_{i}(\zeta)-\left(\nabla J\left(\zeta_{k}\right)+\sum_{i=1}^{m} \lambda_{i} \cdot g_{i}(\zeta)\right)
\end{gathered}
$$

This equation is used to neutralize the gradients between cost function and active constraints, so for balancing, the magnitudes of the Lagrange multipliers are necessary. Positive definiteness of the Hessian matrix is ensured providing $q_{k}^{T} l_{k}$ is positive at each iteration. When $q_{k}^{T} l_{k}$ is not positive, $q_{k}$ is modified on element by element basis so that $q_{k}$ is positive [26].

Quadratic Programming Solution part consists of two steps: first step gives the feasible point and the second step produces iterative process of feasible points to converge 
within the constraints. During this part of nonlinear programming, problem is converted to subproblems and then QP is used to solve, which is formulated as

$$
\begin{aligned}
& \min \frac{1}{2} d^{T} H_{k} d+\nabla J\left(\zeta_{k}\right)^{T} d \\
& \nabla g_{i}\left(\zeta_{k}\right)^{T} d+g_{i}\left(\zeta_{k}\right)=0, i=1,2, \ldots, m_{e} \\
& \nabla g_{i}\left(\zeta_{k}\right)^{T} d+g_{i}\left(\zeta_{k}\right) \leq 0, i=m_{e}+1, \ldots, m
\end{aligned}
$$

The following QP form is used at every iteration.

$$
\min q(d)=\frac{1}{2} d^{T} H d+c^{T} d
$$

subject to following equality and inequality constraints.

$$
\begin{aligned}
& A_{i} d=b_{i}, i=1,2, \ldots, m_{e} \\
& A_{i} d \leq b_{i}, i=m_{e}+1, \ldots, m
\end{aligned}
$$

The solutions of quadratic programming will give $d_{k}$ which is feasible region search direction. Active constraints are updated at every iteration to form a basis for new search direction $d_{k}$.

Optimal Line Search part of the programming is used to produce new iteration using vector $d_{k}$.

$$
\zeta_{k+1}=\zeta_{k}+\alpha d_{k}
$$

where $\alpha$ is the step length parameter, which ensures that $\zeta_{k+1}$ remains in the feasible region.

\section{Controller Design}

Hierarchical control is used as control approach for controller design. PID controllers are used as high level controller to provide virtual command inputs which are used to get reference angles $\left(\phi_{r}, \theta_{r}, \psi_{r}\right)$ with the help of nonlinear optimization. Acquired reference angles are used to produce desired trajectory with the help of the low level controller as shown in closed loop control structure in Fig. 2.

\section{A. Position Control}

From the positional dynamics of quadrotor in (1)

$$
\begin{aligned}
& \mu_{X}=\ddot{X}=(\sin \psi \sin \phi+\cos \psi \sin \theta \cos \phi) \frac{U_{1}}{m} \\
& \mu_{Y}=\ddot{Y}=(-\cos \psi \sin \phi+\sin \psi \sin \theta \cos \phi) \frac{U_{1}}{m} \\
& \mu_{Z}=\ddot{Z}=-g+(\cos \theta \cos \phi) \frac{U_{1}}{m}
\end{aligned}
$$

Errors are defined as

$$
\begin{aligned}
& e_{X}=X_{r}-X \\
& e_{Y}=Y_{r}-Y \\
& e_{Z}=Z_{r}-Z
\end{aligned}
$$

Error dynamics can be formulated as

$$
\begin{aligned}
& \dot{e}_{X}=\dot{X}_{r}-\dot{X} \quad \Rightarrow \ddot{e}_{X}=\ddot{X}_{r}-\ddot{X}=\ddot{X}_{r}-\mu_{X} \\
& \dot{e}_{Y}=\dot{Y}_{r}-\dot{Y} \quad \Rightarrow \ddot{e}_{Y}=\ddot{Y}_{r}-\ddot{Y}=\ddot{Y}_{r}-\mu_{Y} \\
& \dot{e}_{Z}=\dot{Z}_{r}-\dot{Z} \quad \Rightarrow \ddot{e}_{Z}=\ddot{Z}_{r}-\ddot{Z}=\ddot{Z}_{r}-\mu_{Z}
\end{aligned}
$$

$\mu_{X}, \mu_{Y}$ and $\mu_{Z}$ consist of feed forward and feedback terms, which are defined as

$$
\begin{aligned}
& \mu_{X}=\ddot{X}_{r}+K_{p, X} e_{X}+K_{d, X} \dot{e}_{X}+K_{i, X} \int e_{X} d t \\
& \mu_{Y}=\ddot{Y}_{r}+K_{p, Y} e_{Y}+K_{d, Y} \dot{e}_{Y}+K_{i, Y} \int e_{Y} d t \\
& \mu_{Z}=\ddot{Z}_{r}+K_{p, Z} e_{Z}+K_{d, Z} \dot{e}_{Z}+K_{i, Z} \int e_{Z} d t
\end{aligned}
$$

In [15] analytical method was used to calculate the desired attitude angles of the aerial vehicle from desired acceleration vector $(\tau)$ by assuming yaw angle $(\psi)$ to be some fixed value $\left(\psi^{*}\right)$. Desired angles are calculate as

$$
\begin{gathered}
U_{1}=\sqrt{\mu_{X}^{2}+\mu_{Y}^{2}+\left(\mu_{Z}+g\right)^{2}} \\
\theta_{r}=-\arcsin \left(\frac{\mu_{X}}{U_{1}}\right) \\
\phi_{r}=\arcsin \left(\frac{\mu_{Y}}{U_{1} \cos \theta_{r}}\right)
\end{gathered}
$$

\section{B. Attitude Control}

In order to develop controllers for attitude control, from (1) attitude dynamics can be linearized around hover conditions i.e. $\phi, \theta, \psi \approx 0$ and $\dot{\phi}, \dot{\theta}, \dot{\psi} \approx 0$. Angular accelerations in body and world frames will be approximately equal after linearization i.e. $\dot{p} \approx \ddot{\phi}, \dot{q} \approx \ddot{\theta}, \dot{r} \approx \ddot{\psi}$ [15]. Resulting attitude dynamics is given by:

$$
\ddot{\phi}=\frac{U_{2}}{I_{x x}}, \ddot{\theta}=\frac{U_{3}}{I_{y y}}, \ddot{\psi}=\frac{U_{4}}{I_{z z}}
$$

Errors are defined as

$$
e_{\phi}=\phi_{r}-\phi, e_{\theta}=\theta_{r}-\theta, e_{\psi}=\psi_{r}-\psi
$$

Similarly error dynamics are defined as

$$
\begin{aligned}
& \dot{e}_{\phi}=\dot{\phi}_{r}-\dot{\phi} \Rightarrow \ddot{e}_{\phi}=\ddot{\phi}_{r}-\ddot{\phi} \\
& \dot{e}_{\theta}=\dot{\theta}_{r}-\dot{\theta} \Rightarrow \ddot{e}_{\theta}=\ddot{\theta}_{r}-\ddot{\theta} \\
& \dot{e}_{\psi}=\dot{\psi}_{r}-\dot{\psi} \Rightarrow \ddot{e}_{\psi}=\ddot{\psi}_{r}-\ddot{\psi}
\end{aligned}
$$

Attitude controllers are designed as

$$
\begin{aligned}
& U_{2}=I_{x x}\left(\ddot{\phi}_{r}+K_{p, \phi} e_{\phi}+K_{d, \phi} \dot{e}_{\phi}+K_{i, \phi} \int e_{\phi} d t\right) \\
& U_{3}=I_{y y}\left(\ddot{\theta}_{r}+K_{p, \theta} e_{\theta}+K_{d, \theta} \dot{e}_{\theta}+K_{i, \theta} \int e_{\theta} d t\right) \\
& U_{4}=I_{z z}\left(\ddot{\psi}_{r}+K_{p, \psi} e_{\psi}+K_{d, \psi} \dot{e}_{\psi}+K_{i, \psi} \int e_{\psi} d t\right)
\end{aligned}
$$

\section{Simulation Results}

A control allocation type approach has been implemented where reference angles are generated through numerical method using nonlinear optimization. Analytical method for reference angles calculation is also considered for comparison. For simulation, the following two scenarios are taken into account.

1) During the first scenario yaw angle $(\psi)$ is fixed to a constant value $\left(\psi^{*}\right)$ for both numerical and analytical methods. 


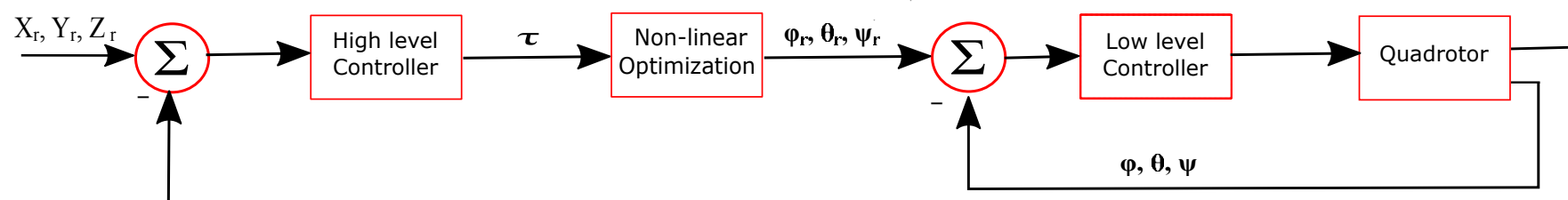

$\mathrm{X}, \mathrm{Y}, \mathrm{Z}$

Fig. 2: Closed Loop Control System

2) During the second scenario, numerical method has been taken into account and yaw angle $(\psi)$ is not fixed and constraints have been put on all reference angles.

For nonlinear optimization, stopping criterion specifications are summarized in the TABLE I.

TABLE I: Nonlinear Optimization Specifications

\begin{tabular}{|l|c|}
\hline Specifications & Range \\
\hline Max Iterations & 400 \\
\hline Function Tolerance & $1 \mathrm{e}-06$ \\
\hline Constraint Tolerance & $1 \mathrm{e}-06$ \\
\hline Max Function Evaluations & 100 \\
\hline First Order Optimality Measure & $1 \mathrm{e}-06$ \\
\hline
\end{tabular}

For trajectory it is assumed that quadrotor takesoff vertically from the spot along the $z$ axis, then hovers at desired altitude, takes a straight flight along the $x$ axis, moves in a circular loop and lands at the desired spot after taking some more straight flight. Trajectory has been shown in Figure 3.

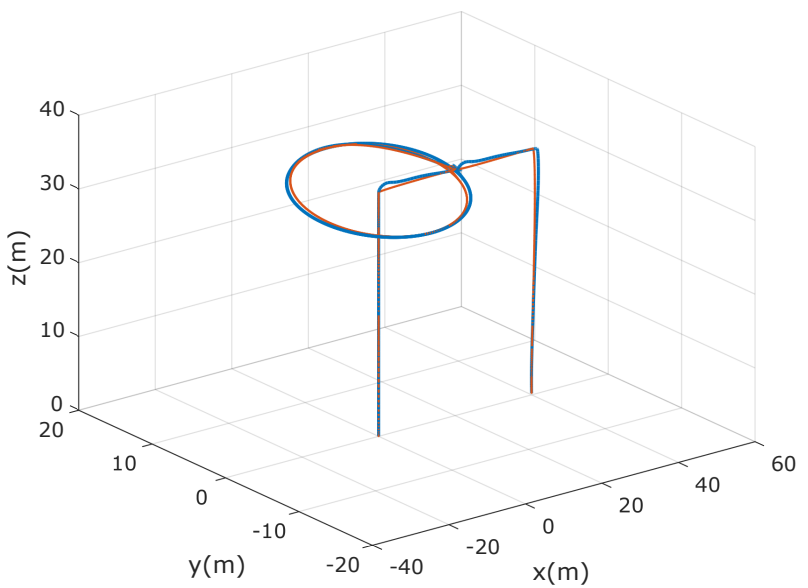

Fig. 3: Trajectory Tracking (Desired=red, Actual=blue--)

During the first scenario of the simulation yaw angle $(\psi)$ is taken to be fixed at $3.5^{\circ}$ for the calculation of reference angles through analytical and numerical method. Cartesian positions of the quadrotor during the trajectory for both methods have been shown in Figure 4 which shows that quadrotor successfully tracked the desired trajectory for both methods.

Position errors in Figure 5 show the efficiency of tracking for the numerical method. It has been observed that cartesian
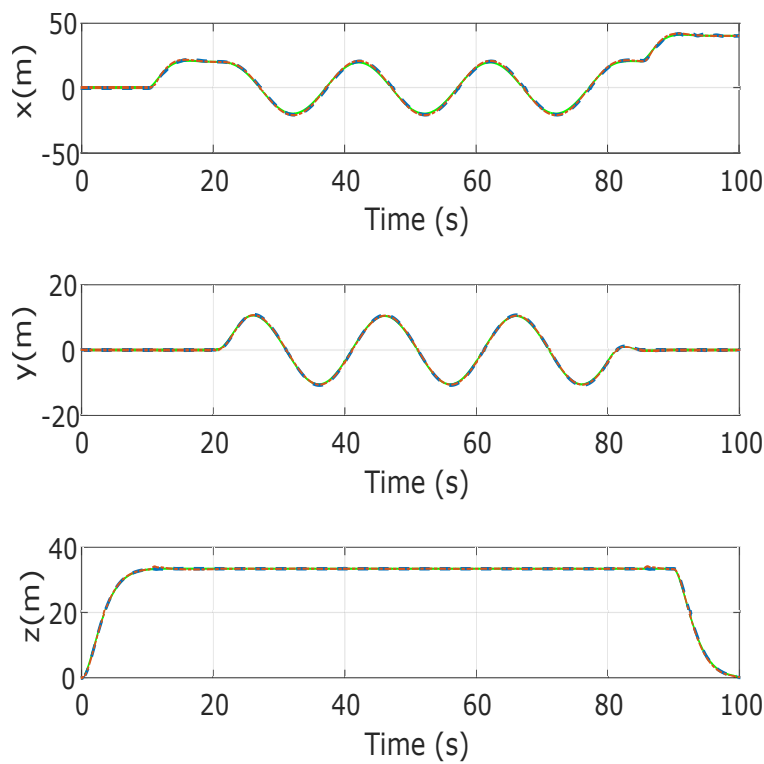

Fig. 4: Cartesian positions of the quadrotor vs Time (Desired $=$ green, Analytical $=$ blue -- , Numerical $=$ red - .)
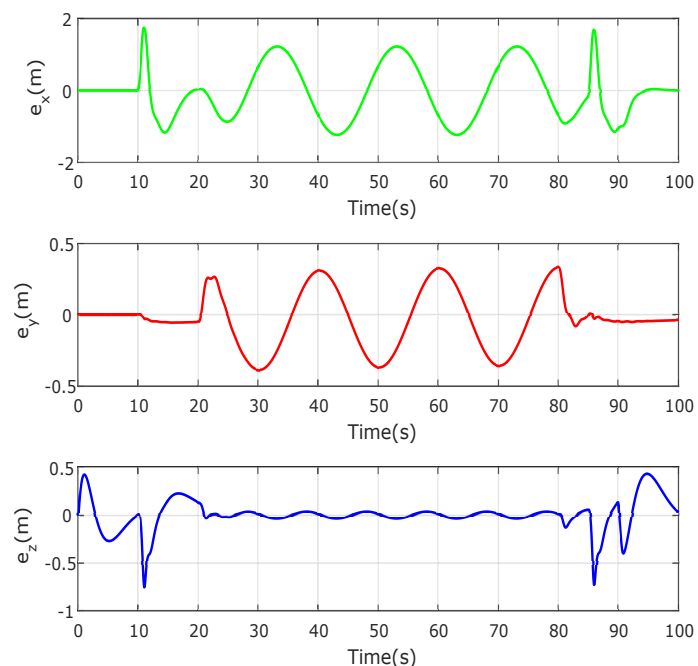

Fig. 5: Position Errors

positions of the quadrotor demonstrate similar behavior during the circular path but during the transition from hovering 
TABLE II: Euler Angles and Total Thrust $\left(U_{1}\right)$ Bound Values

\begin{tabular}{|c|c|c|}
\hline Euler Angles & Range & Rate \\
\hline$\phi$ & -14 to $14(\mathrm{deg})$ & $\leq 8.5(\mathrm{deg} / \mathrm{sample})$ \\
\hline$\theta$ & -14 to $14(\mathrm{deg})$ & $\leq 8.5(\mathrm{deg} / \mathrm{sample})$ \\
\hline$\psi$ & -7 to $7(\mathrm{deg})$ & $\leq 5.5(\mathrm{deg} / \mathrm{sample})$ \\
\hline$U_{1}$ & 0 to 20 & $\leq 10$ \\
\hline
\end{tabular}

state to straight flight, numerical method showed smoother results than analytical method of finding reference Euler angles which can be seen during 10 to 20 seconds and after 85 seconds in Figure 6.
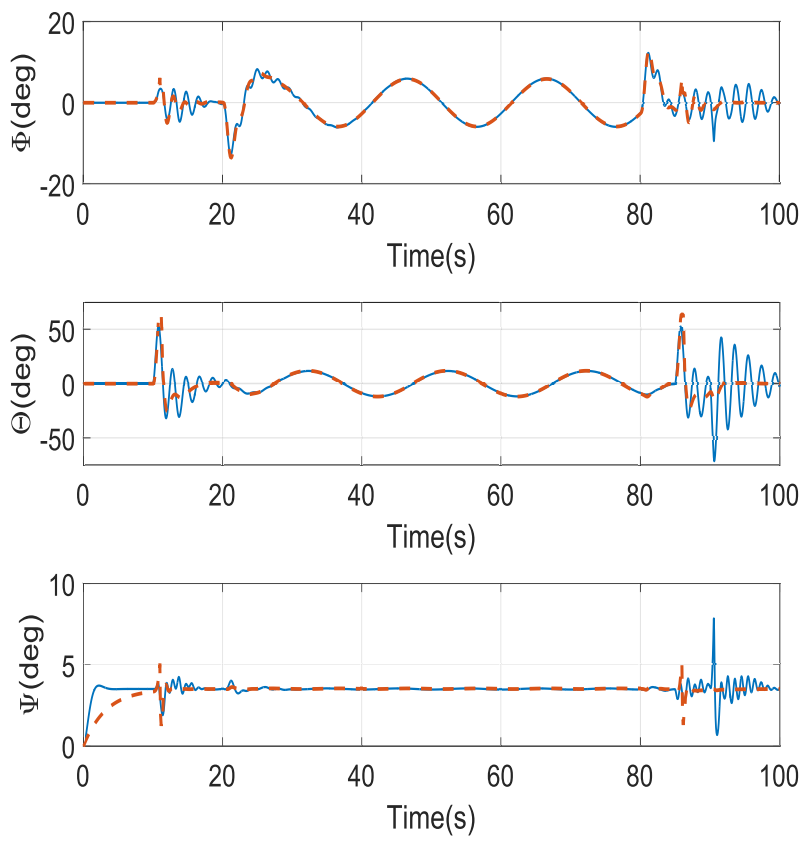

Fig. 6: Euler Angles (Analytical=solid, Numerical=dashed)

In the second scenario, yaw angle $(\psi)$ is not taken to be fixed; instead constraints have been put on all reference angles which are given in TABLE II. Simulation results for trajectory curves have been shown for constrained optimization in Figure 7, which shows that quadrotor successfully tracked the trajectory. In Figure 8 comparison of Euler angles for constrained optimization in the first scenario when the yaw angle $(\psi)$ is fixed and for constraint optimization for range of values for all reference angles including yaw angle $(\psi)$, shows that Euler angles not only change in a smooth fashion but also remain within the bounds for the same trajectory. In Figure 9 control efforts for numerical method when yaw angle $(\psi)$ is not fixed are plotted and it is clear that each one of them stays withing phsyical bounds.

\section{CONCLUSION AND FUtURE Work}

In this paper, a control allocation like method using nonlinear constraint optimization is implemented on the nonlinear dynamics of the quadrotor, which consists of high level control and low level control. High level controller is used to get necessary desired generalized command signals. Nonlinear constraint optimization is used at every instant to get the
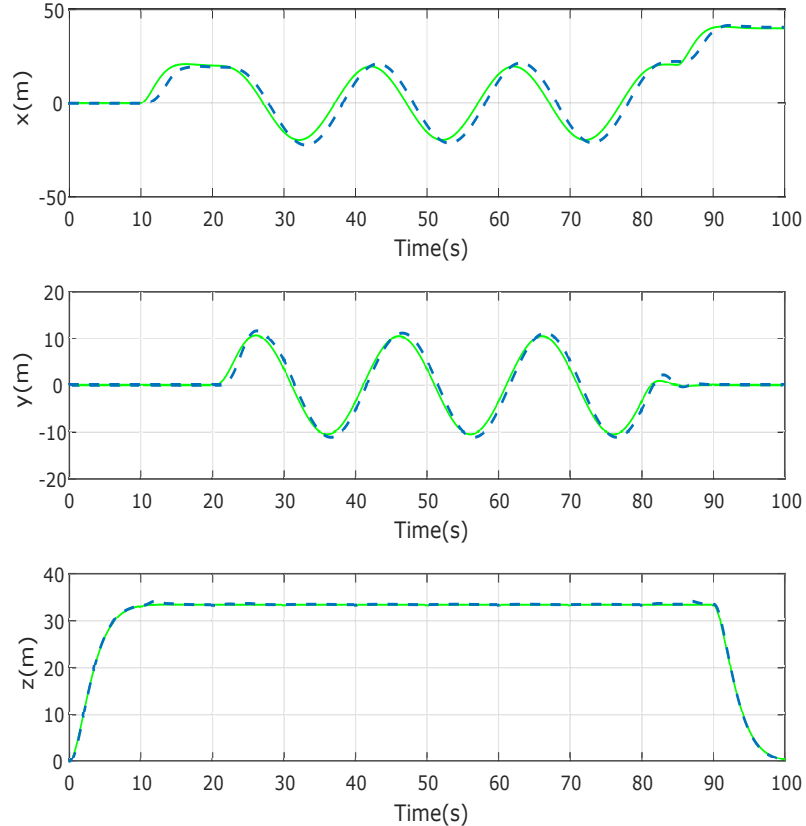

Fig. 7: Cartesian positions of the quadrotor vs Time for bounded angles optimization (Desired=solid, Actual=dashed)
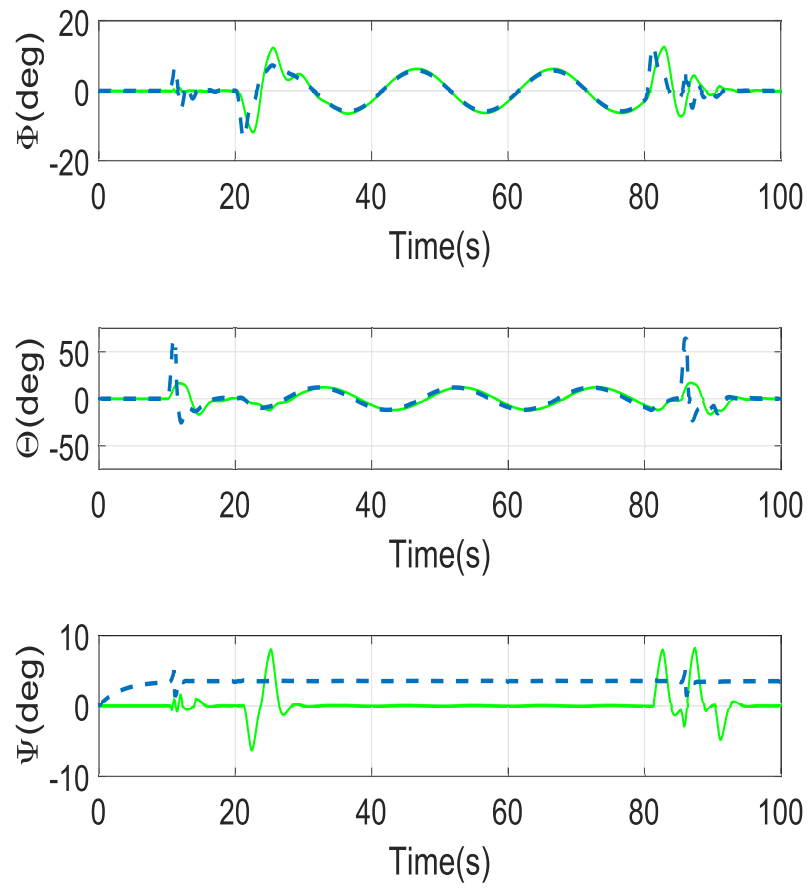

Fig. 8: Euler Angles (For fixed psi $\left(\psi^{\star}\right)=$ dashed, for bounded values of psi $(\psi)=$ solid))

reference angles for the desired trajectory tracking according to generalized commands. Low level controllers are used for attitude control. Hierarchical control allows us to design separately the controllers for position and attitude for highly coupled nonlinear dynamics of quadrotor. For trajectory, it has been considered that vehicle moves in a circular loop after hovering at the desired altitude. For trajectory tracking, two scenarios have been taken into account based on yaw 

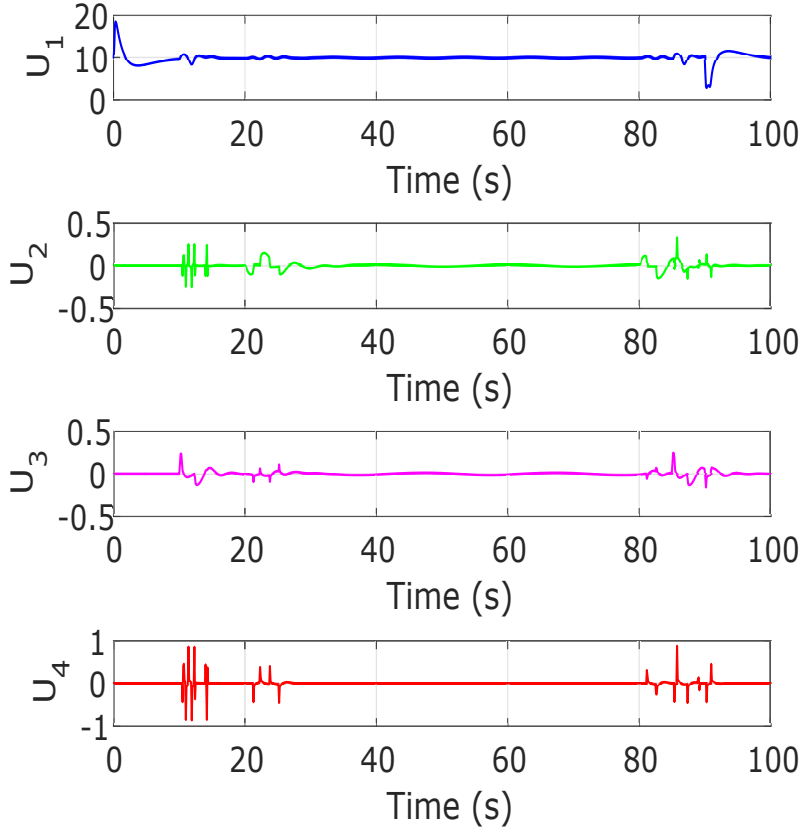

Fig. 9: Control Efforts

angle $(\psi)$. Simulation results for both scenarios have been shown for both analytical and numerical methods of finding reference angles for comparison. From results it has been inferred that both methods provided successful trajectory tracking but numerical method showed smoother changes in Euler angles than the analytical method. Simulation results have also been presented for the second scenario when the yaw angle is not taken as fixed; instead bounded constraints have been considered for optimization. Results showed that quadrotor successfully tracked the trajectory with the desired attitude angles remaining within the bounds for the whole time period.

As future work, we will work on making the proposed technique real-time and then test it on a physical quadrotor.

\section{REFERENCES}

[1] S. Bouabdallah, P. Murrieri, and R. Siegwart, "Design and Control of an Indoor Micro Quadrotor,"Proceedings of the 2004 IEEE International Conference on Robotics \& Automation, New Orleans, LA, 2004, pp. 43934398.

[2] A. Mokhtari and A. Benallegue,"Dynamic Feedback Controller of Euler Angles and Wind Parameters Estimation for a Quadrotor Unmanned Aerial Vehicle,"Proceedings of the 2004 IEEE International Conference on Robotics \& Automation, New Orleans, LA, 2004, pp. 23592366.

[3] S. Bouabdallah and R. Siegwart, "Backstepping and Sliding Mode Techniques Applied to an Indoor Micro Quadrotor,"Proceedings of the 2005 IEEE. International Conference on Robotics \& Automation, Barcelona, Spain, April 2005, pp. 22592264.

[4] H. Bouadi, M. Bouchoucha, and M. Tadjine,"Sliding Mode Control Based on Backstepping Approach for an UAV TypeQuadrotor,'International. Journal. Of Applied Math. \& Computer Sciences, vol.4, no.2, 2007.

[5] G. M. Hoffmann, H. Huang, S. L. Waslander, and C. J. Tomlin, "Quadrotor Helicopter Light Dynamics and Control: Theory and Experiment,"AIAA Guidance Navigation and Control Conference and Exhibit, no. AIAA-2007-6461, Hilton Head, South Carolina, Aug. 2007.
[6] I. D. Cowling, O. A. Yakimenko, J. F. Whidborne, and A. K Cooke, "A Prototype of an Autonomous Controller for a Quadrotor UAV,'European Control Conference, 2007, pp. 18.

[7] D. Lee, H. J. Kim, and S. Sastry, "Feedback Linearization vs Adaptive Sliding Mode Control for a Quadrotor Helicopter,'International Journal of Control, Automation and Systems, vol. 7, no. 3, pp. 419428 , 2009.

[8] T. Madani and A. Benallegue, "Adaptive Control via Backstepping Technique and Neural Networks of a Quadrotor Helicopter,'Proceedings of the 17th World Congress of IFAC, Seoul, Korea, July 2008, pp. 65136518.

[9] F. Yacef, O. Bouhali and M. Hamerlain, "Adaptive Fuzzy Backstepping Control for Trajectory Tracking of Unmanned Aerial Quadrotor,"International Conference on Unmanned Aircraft Systems (ICUAS), May 27-30, 2014. Orlando, FL, USA.

[10] K. Benzaid, N. Mansouri and O. L. Igbida, "Robust Trajectory Tracking Control of a Quadrotor", Proceedings of the 3rd International Conference on Systems and Control, Algiers, Algeria, October 29-31, 2013.

[11] A. Abdessameud and A. Tayebi, "Global Trajectory Tracking Control of VTOL-UAVs without Linear Velocity Measurements", Automatica, vol. 46, no. 6, pp. 10531059, June 2010.

[12] S. Formentin and M. Lovera, "Flatness-Based Control of a Quadrotor Helicopter via Feedforward Linearization,'IEEE Conference on Decision and Control and European Control Conference (CDC-ECC), Orlando, FL, USA, 2011, pp. 61716176.

[13] G. V. Raffo, Manuel G. Ortega Francisco, R. Rubio "MPC with Nonlinear $H \propto$ Control for Path Tracking of a Quadrotor Helicopter", Proceedings of the 17th World Congress, the International Federation of Automatic Control, Seoul, Korea, July 6-11, 2008.

[14] E. Cetinsoy, S. Dikyar, C. Hancer, K.T. Oner, E. Sirimoglu, M. Unel, M.F. Aksit, "Design and Construction of a Novel quad Tilt-Wing UAV", Mechatronics, Volume 22, Issue 6, September 2012, Pages 723745 .

[15] C. Hancer, K. T. Oner, E. Sirimoglu, E.Cetinsoy, M. Unel, "Robust Position Control of a Tilt-Wing Quadrotor",49th IEEE Conference on Decision and Control, December 15-17, 2010 Hilton Atlanta Hotel, Atlanta, GA, USA.

[16] C. Hancer, K. T. Oner, E. Sirimoglu, E. Cetinsoy, M. Unel, "Robust Hovering Control of a Quad Tilt-Wing UAV", in Proc. 36th Annual Conference on IEEE Industrial Electronics Society Glendale, AZ, Nov. 2010, pp. 16151620.

[17] Y. Yildiz, M. Unel and A. E. Demirel, "Adaptive Nonlinear Hierarchical Control of a Quad Tilt-Wing UAV”, European Control Conference (ECC), July 15-17, 2015. Linz, Austria.

[18] Y. Yildiz, M. Unel, A. E. Demirel, "Nonlinear Hierarchical Control of a Quad-Tilt-Wing UAV: An Adaptive Control Approach", International Journal of Adaptive Control and Signal Processing, DOI:10.1002/acs2759, 2017.

[19] H. L. Chan and K. T. Woo, "Design and Control of Small Quadcopter System with Motor Closed Loop Speed Control", International Journal of Mechanical Engineering and Robotics Research, vol. 4, no.1, October 2015.

[20] Y. Bouktir, M. Haddad, T. Chettibi ,"Trajectory Planning for a Quadrotor Helicopter", 16th Mediterranean Conference on Control and Automation Congress Centre, Ajaccio, France June 25-27, 2008.

[21] J. C. Monteiro, F. Lizarralde, L Hsu, "Optimal Control Allocation of Quadrotor UAVs subject to Actuator Constraints ", American Control Conference (ACC), Boston, MA, USA.

[22] T. A. Johansen, T. I. Fossen, and S. P. Berge, "Constrained Nonlinear Control Allocation With Singularity Avoidance Using Sequential Quadratic Programming", IEEE Transactions On Control Systems Technology, vol.12, no.1, January 2004.

[23] T. Bresciani, Modelling, "'Identification and Control of a Quadrotor", Helicopter, MSc thesis, Department of Automatic Control Lund University, Sweden.

[24] J. Hewitt, "Drone Athleticism: Astounding New quadrotor control, and Beyond", June 14, 2013. Retrieved from https://www.extremetech.com/extreme/158510-drone-athleticismastounding-new-quadrotor-control-and-beyond

[25] T. A. Johansen, T. I. Fossen (2013), "Control Allocation-A Survey ", Automatica, Volume 49, Issue 5, Pages 10871103, May 2013.

[26] T. Coleman, M. A. Branch, A. Grace,"Optimization Toolbox, For Use with Matlab ", Mathworks, 1999. 